\title{
A new fractional SIRS-SI malaria disease model with application of vaccines, antimalarial drugs, and spraying
}

\author{
Devendra Kumar ${ }^{1 *}$, Jagdev Singh², Maysaa Al Qurashi and Dumitru Baleanu ${ }^{4,5}$
}

"Correspondence:

devendra.maths@gmail.com ${ }^{1}$ Department of Mathematics, University of Rajasthan, Jaipur, India Full list of author information is available at the end of the article

\begin{abstract}
The present paper deals with a new fractional SIRS-SI model describing the transmission of malaria disease. The SIRS-SI malaria model is modified by using the Caputo-Fabrizio fractional operator for the inclusion of memory. We also suggest the utilization of vaccines, antimalarial medicines, and spraying for the treatment and control of the malaria disease. The theory of fixed point is utilized to examine the existence of the solution of a fractional SIRS-SI model describing spreading of malaria. The uniqueness of the solution of SIRS-SI model for malaria is also analyzed. It is shown that the treatments have great impact on the dynamical system of human and mosquito populations. The numerical simulation of fractional SIRS-SI malaria model is performed with the aid of HATM and Maple packages to show the effect of different parameters of the treatment of malaria disease. The numerical results for fractional SIRS-SI malaria model reveal that the recommended approach is very accurate and effective.
\end{abstract}

Keywords: Fractional SIRS-SI malaria model; Fixed point theorem; Caputo-Fabrizio fractional operator; HATM

\section{Introduction}

Malaria is a life-threatening mosquito-borne blood illness in the developing portion of the globe and especially in Asia and Africa. It is caused by a plasmodium parasite. The reports by the WHO show that malaria is a major risk to human life and remains a high-risk infectious illness. The financial load on the infected areas by malaria disease is massive and requires serious public health attention. Mathematical modeling of infectious diseases is a very strong tool to understand the dynamical system of disease spreading and control strategies. Recently many scientists and mathematicians have suggested mathematical models for malaria transmission. Agusto et al. [1] reported a SIR having infection rate of nonlinear type with the aid of vaccination for the human population. Abdullahi et al. [2] analyzed the outcomes of treatment as a control variable on the malaria transmission process. Mandal et al. [3] formulated the mathematical representation of malaria transmission with the situation of person-to-person transmission via blood transfusions and malaria-infected ladies having pregnancy. Chiyaka et al. [4] suggested a modified mathematical modeling by assuming that the persons also belonging to the recov-

(c) The Author(s) 2019. This article is distributed under the terms of the Creative Commons Attribution 4.0 International License (http://creativecommons.org/licenses/by/4.0/), which permits unrestricted use, distribution, and reproduction in any medium, provided you give appropriate credit to the original author(s) and the source, provide a link to the Creative Commons license, and indicate if changes were made. 
ered group have a probability to be susceptible. Rafikov et al. [5] reported an efficient control strategy of malaria vector with the aid of genetically altered mosquitoes. Yang [6] suggested another approach for describing the malaria transmission connected with global warming and local socioeconomic circumstances. In a recent work, Senthamarai et al. [7] utilized the HAM to examine the spreading of malaria illness in an SIRS-SI model. However, all these approaches and mathematical models have their own limitation due to local nature of integer-order derivatives. Therefore fractional derivative approaches are suggested in mathematical modeling of biological and physical systems [821].

Very recently, Caputo and Fabrizio [22] reported a novel operator namely the CaputoFabrizio (CF) fractional operator involving a nonsingular kernel. Furthermore, the additional properties of this operator were put up by Losada and Nieto [23]. The suitability and efficiency of the CF fractional operator have been demonstrated by many researchers. Singh et al. [24] reported a mathematical model for computer viruses incorporating the CF fractional operator. Singh et al. [25] suggested an innovative idea for mathematical modeling of giving up smoking dynamics with the aid of the CF fractional operator. Djida and Atangana [26] studied a water flow within a confined aquifer connected to an arbitrary order operator in the terms of the Caputo-Fabrizio and many others. Inspired by ongoing investigations on the CF fractional derivative and their effectiveness, we employ this derivative in SIRS-SI malaria model for inclusion of memory. The existence and uniqueness of the solution of SIRS-SI model describing spreading of malaria involving memory effects is shown by applying the fixed-point theory. The numerical computation for the fractional SIRS-SI malaria model is executed by using HATM [27-31] and Padé approximation [32]. The present paper is developed as follows. In Sect. 2, we present the required results pertaining to the CF operator of arbitrary order. In Sect. 3, we give a mathematical formulation of fractional SIRS-SI malaria model. Section 4 concerns with the existence and uniqueness analysis of solution of SIRSSI model representing the malaria having fractional order. In Sect. 5, we use the efficiency of HATM for examining fractional SIRS-SI malaria model. In Sect. 6, we investigate the effect of various parameters on humans and mosquitoes. Finally, in Sect. 7, we conclude.

\section{Preliminaries}

Definition 1 Let $\psi \in H^{1}\left(\ell_{1}, \ell_{2}\right), \ell_{2}>\ell_{1}, \lambda \in(0,1]$. Then the CF fractional operator [22] is expressed as

$$
\begin{aligned}
D_{t}^{\lambda}(\psi(t)) & =\frac{M(\lambda)}{1-\lambda} \int_{\ell_{1}}^{t} \psi^{\prime}(\theta) \exp \left[-\lambda \frac{t-\theta}{1-\lambda}\right] d \theta, \quad 0<\lambda<1, \\
& =\frac{d \psi}{d t}, \quad \lambda=1 .
\end{aligned}
$$

In this expression $M(\lambda)$ satisfies the condition $M(0)=M(1)=1[22]$.

Definition 2 The integral operator of fractional order corresponding to the CF fractional derivative is defined as [23]

$$
I_{t}^{\lambda}(\psi(t))=\frac{2(1-\lambda)}{(2-\lambda) M(\lambda)} \psi(t)+\frac{2 \lambda}{(2-\lambda) M(\lambda)} \int_{0}^{t} \psi(\varsigma) d \varsigma, \quad t \geq 0 .
$$


Definition 3 The Laplace transform of ${ }_{0}^{C F} D_{t}^{\lambda} \psi(t)$ is represented as

$$
L\left[{ }_{0}^{C F} D_{t}^{\lambda} \psi(t)\right]=M(\lambda) \frac{s L[\psi(t)]-\psi(0)}{s+\lambda(1-s)} .
$$

\section{Fractional SIRS-SI malaria model with exponential law}

We suppose that the population of human is categorized into the following three groups:

Group I: Susceptible human denoted by $S_{h}$;

Group II: Infected human denoted by $I_{h}$;

Group III: Recovered human denoted by $R_{h}$.

The population of mosquito is categorized into the following two groups:

Group I: Susceptible mosquito denoted by $S_{m}$;

Group II: Infected mosquito denoted by $I_{m}$.

Let us suppose that the person who is born is transferred to the susceptible group with fixed rate $\alpha_{h}$ per unit time. Peoples in the susceptible group are transferred to the infected group because of blood exchanging with rate $\omega \gamma_{1}$ per unit time (here $\omega$ indicates the average number of blood transfusions between the susceptible and infected groups in a fixed time period, whereas $\gamma_{1}$ stands for the probability of disease transfer from an infected person to a susceptible person) or through an infected mosquito bite with rate $\xi \gamma_{2}$ per unit time (here $\xi$ stands for the average number of infected mosquito bites on a susceptible person in a fixed time period, whereas $\gamma_{2}$ stands for the probability of disease transfer to susceptible persons through infected mosquitoes). Persons in the susceptible group transfer into the recovered group because of vaccination with rate $\delta$ per unit time. The persons in the susceptible group expire with rate $\eta_{h}$. A newly born baby is infected by malaria because of inbred at rate $\mu$ per unit time. The persons in infected group can transfer to the recovered group because of using antimalarial drugs at rate $c v$ per unit time (here $c$ stands for the rate of people healing, and $v$ indicates the potency of antimalarial medicines). Peoples in the infected group can expire with rate $\eta_{h}$ and die because of malaria disease with rate $\varepsilon$ per unit time. Peoples in recovered group die with rate $\eta_{h}$ per unit time. Moreover, mosquitoes are born and transferred to the susceptible group at a fixed rate $\alpha_{m}$ per unit time. The mosquitoes in the susceptible group can transfer into the infected group by biting of mosquito to the infected persons with rate $e \gamma_{3}$ per unit time (here $e$ stand for the average number of susceptible mosquito bites to the infected persons in a fixed time period, and $\gamma_{3}$ indicates the possibility of disease passing to susceptible mosquitoes from the infected peoples) or can expire with rate $\eta_{m}$ per unit time. The average per capita rate of loss of immunity is $\beta$ per unit time. The mosquitoes belonging to the susceptible and infected groups can expire due to exercise of spraying with rate $\sigma$ per unit time. The mosquitoes belonging to the infected group can die with rate $\eta_{m}$ per unit time. The governing equations for the SIRS-SI malaria model are presented 
as follows:

$$
\begin{aligned}
& \frac{d S_{h}}{d t}=\alpha_{h}+\beta R_{h}-\left(\omega \gamma_{1} I_{h}+\xi \gamma_{2} I_{m}\right) S_{h}-\left(\delta+\eta_{h}\right) S_{h}, \\
& \frac{d I_{h}}{d t}=\mu I_{h}+\left(\omega \gamma_{1} I_{h}+\xi \gamma_{2} I_{m}\right) S_{h}-\left(\eta_{h}+\varepsilon+c \nu\right) I_{h} \\
& \frac{d R_{h}}{d t}=c \nu I_{h}-\left(\eta_{h}+\beta\right) R_{h}+\delta S_{h} \\
& \frac{d S_{m}}{d t}=\alpha_{m}-\left(e \gamma_{3} I_{h}+\eta_{m}+\sigma\right) S_{m} \\
& \frac{d I_{m}}{d t}=e \gamma_{3} I_{h} S_{m}-\left(\eta_{m}+\sigma\right) I_{m} .
\end{aligned}
$$

Since the integer-order derivative is local in nature, the presented SIRS-SI malaria model (4) does not describe different effects on humans and mosquitoes in efficient manner. Therefore, to include the memory effects in the description of malaria disease, we extend the model (4) by employing the newly proposed Caputo-Fabrizio fractional derivative as follows:

$$
\begin{aligned}
& { }^{C F} D_{0}^{\lambda} S_{h}=\alpha_{h}+\beta R_{h}-\left(\omega \gamma_{1} I_{h}+\xi \gamma_{2} I_{m}\right) S_{h}-\left(\delta+\eta_{h}\right) S_{h}, \\
& { }^{C F} D_{0}^{\lambda} I_{h}=\mu I_{h}+\left(\omega \gamma_{1} I_{h}+\xi \gamma_{2} I_{m}\right) S_{h}-\left(\eta_{h}+\varepsilon+c v\right) I_{h}, \\
& { }^{C F} D_{0}^{\lambda} R_{h}=c v I_{h}-\left(\eta_{h}+\beta\right) R_{h}+\delta S_{h}, \\
& { }^{C F} D_{0}^{\lambda} S_{m}=\alpha_{m}-\left(e \gamma_{3} I_{h}+\eta_{m}+\sigma\right) S_{m}, \\
& { }^{C F} D_{0}^{\lambda} I_{m}=e \gamma_{3} I_{h} S_{m}-\left(\eta_{m}+\sigma\right) I_{m},
\end{aligned}
$$

with the initial conditions

$$
S_{h}(0)=c_{1}, \quad I_{h}(0)=c_{2}, \quad R_{h}(0)=c_{3}, \quad S_{m}(0)=c_{4}, \quad I_{m}(0)=c_{5} .
$$

Let us suppose that $B$ is the Banach space of continuous real-valued functions defined on an interval $I$ with the associated norm

$$
\left\|\left(S_{h}, I_{h}, R_{h}, S_{m}, I_{m}\right)\right\|=\left\|S_{h}\right\|+\left\|I_{h}\right\|+\left\|R_{h}\right\|+\left\|S_{m}\right\|+\left\|I_{m}\right\| .
$$

In Eq. (7), we have $\left\|S_{h}\right\|=\sup \left\{\left|S_{h}(t)\right|: t \in I\right\},\left\|I_{h}\right\|=\sup \left\{\left|I_{h}(t)\right|: t \in I\right\},\left\|R_{h}\right\|=\sup \left\{\left|R_{h}(t)\right|:\right.$ $t \in I\},\left\|S_{m}\right\|=\sup \left\{\left|S_{m}(t)\right|: t \in I\right\}$, and $\left\|I_{m}\right\|=\sup \left\{\left|I_{m}(t)\right|: t \in I\right\}$. Specifically, $B=E(I) \times$ $E(I) \times E(I) \times E(I) \times E(I)$, where $E(I)$ stands for the Banach space of continuous real-valued functions on $I$ and the associated sup norm.

\section{Existence and uniqueness analysis}

This section deals with the existence and uniqueness analysis of the solution of the fractional SIRS-SI malaria model with exponential law. It is very important to know about the existence and uniqueness of the solution of any mathematical model in natural sciences. Therefore we examine the existence and uniqueness of the solution of fractional SIRS-SI malaria model by using fixed point theory [33-35]. 
We apply the fractional integral operator (2) to Eq. (5), which gives

$$
\begin{aligned}
& S_{h}(t)-S_{h}(0)={ }_{0}^{C F} I_{t}^{\lambda}\left\{\alpha_{h}+\beta R_{h}-\left(\omega \gamma_{1} I_{h}+\xi \gamma_{2} I_{m}\right) S_{h}-\left(\delta+\eta_{h}\right) S_{h}\right\}, \\
& I_{h}(t)-I_{h}(0)={ }_{0}^{C F} I_{t}^{\lambda}\left\{\mu I_{h}+\left(\omega \gamma_{1} I_{h}+\xi \gamma_{2} I_{m}\right) S_{h}-\left(\eta_{h}+\varepsilon+c \nu\right) I_{h}\right\}, \\
& R_{h}(t)-R_{h}(0)={ }_{0}^{C F} I_{t}^{\lambda}\left\{c \nu I_{h}-\left(\eta_{h}+\beta\right) R_{h}+\delta S_{h}\right\}, \\
& S_{m}(t)-S_{m}(0)={ }_{0}^{C F} I_{t}^{\lambda}\left\{\alpha_{m}-\left(e \gamma_{3} I_{h}+\eta_{m}+\sigma\right) S_{m}\right\}, \\
& I_{m}(t)-I_{m}(0)={ }_{0}^{C F} I_{t}^{\lambda}\left\{e \gamma_{3} I_{h} S_{m}-\left(\eta_{m}+\sigma\right) I_{m}\right\} .
\end{aligned}
$$

Using the notation suggested in [23], we have

$$
\begin{aligned}
& S_{h}(t)-S_{h}(0) \\
& =\frac{2(1-\lambda)}{(2-\lambda) M(\lambda)}\left\{\alpha_{h}+\beta R_{h}(t)-\left(\omega \gamma_{1} I_{h}(t)+\xi \gamma_{2} I_{m}(t)\right) S_{h}(t)-\left(\delta+\eta_{h}\right) S_{h}(t)\right\} \\
& +\frac{2 \lambda}{(2-\lambda) M(\lambda)} \int_{0}^{t}\left\{\alpha_{h}+\beta R_{h}(\varsigma)-\left(\omega \gamma_{1} I_{h}(\varsigma)+\xi \gamma_{2} I_{m}(\varsigma)\right) S_{h}(\varsigma)\right. \\
& \left.-\left(\delta+\eta_{h}\right) S_{h}(\varsigma)\right\} d \varsigma, \\
& I_{h}(t)-I_{h}(0) \\
& =\frac{2(1-\lambda)}{(2-\lambda) M(\lambda)}\left\{\mu I_{h}(t)+\left(\omega \gamma_{1} I_{h}(t)+\xi \gamma_{2} I_{m}(t)\right) S_{h}(t)-\left(\eta_{h}+\varepsilon+c \nu\right) I_{h}(t)\right\} \\
& +\frac{2 \lambda}{(2-\lambda) M(\lambda)} \int_{0}^{t}\left\{\mu I_{h}(\varsigma)+\left(\omega \gamma_{1} I_{h}(\varsigma)+\xi \gamma_{2} I_{m}(\varsigma)\right) S_{h}(\varsigma)\right. \\
& \left.-\left(\eta_{h}+\varepsilon+c \nu\right) I_{h}(\varsigma)\right\} d \varsigma, \\
& R_{h}(t)-R_{h}(0) \\
& =\frac{2(1-\lambda)}{(2-\lambda) M(\lambda)}\left\{c v I_{h}(t)-\left(\eta_{h}+\beta\right) R_{h}(t)+\delta S_{h}(t)\right\} \\
& +\frac{2 \lambda}{(2-\lambda) M(\lambda)} \int_{0}^{t}\left\{c v I_{h}(\varsigma)-\left(\eta_{h}+\beta\right) R_{h}(\varsigma)+\delta S_{h}(\varsigma)\right\} d \varsigma, \\
& S_{m}(t)-S_{m}(0) \\
& =\frac{2(1-\lambda)}{(2-\lambda) M(\lambda)}\left\{\alpha_{m}-\left(e \gamma_{3} I_{h}(t)+\eta_{m}+\sigma\right) S_{m}(t)\right\} \\
& +\frac{2 \lambda}{(2-\lambda) M(\lambda)} \int_{0}^{t}\left\{\alpha_{m}-\left(e \gamma_{3} I_{h}(\varsigma)+\eta_{m}+\sigma\right) S_{m}(\varsigma)\right\} d \varsigma, \\
& I_{m}(t)-I_{m}(0) \\
& =\frac{2(1-\lambda)}{(2-\lambda) M(\lambda)}\left\{e \gamma_{3} I_{h}(t) S_{m}(t)-\left(\eta_{m}+\sigma\right) I_{m}(t)\right\} \\
& +\frac{2 \lambda}{(2-\lambda) M(\lambda)} \int_{0}^{t}\left\{e \gamma_{3} I_{h}(\varsigma) S_{m}(\varsigma)-\left(\eta_{m}+\sigma\right) I_{m}(\varsigma)\right\} d \varsigma .
\end{aligned}
$$


For clarity, we express

$$
\begin{aligned}
& \Omega_{1}\left(t, S_{h}\right)=\alpha_{h}+\beta R_{h}(t)-\left(\omega \gamma_{1} I_{h}(t)+\xi \gamma_{2} I_{m}(t)\right) S_{h}(t)-\left(\delta+\eta_{h}\right) S_{h}(t), \\
& \Omega_{2}\left(t, I_{h}\right)=\mu I_{h}(t)+\left(\omega \gamma_{1} I_{h}(t)+\xi \gamma_{2} I_{m}(t)\right) S_{h}(t)-\left(\eta_{h}+\varepsilon+c \nu\right) I_{h}(t), \\
& \Omega_{3}\left(t, R_{h}\right)=c \nu I_{h}(t)-\left(\eta_{h}+\beta\right) R_{h}(t)+\delta S_{h}(t), \\
& \Omega_{4}\left(t, S_{m}\right)=\alpha_{m}-\left(e \gamma_{3} I_{h}(t)+\eta_{m}+\sigma\right) S_{m}(t) \\
& \Omega_{5}\left(t, I_{m}\right)=e \gamma_{3} I_{h}(t) S_{m}(t)-\left(\eta_{m}+\sigma\right) I_{m}(t) .
\end{aligned}
$$

Theorem 1 The kernels $\Omega_{1}, \Omega_{2}, \Omega_{3}, \Omega_{4}$, and $\Omega_{5}$ satisfy the Lipschitz condition and contraction if

$$
0 \leq \omega \gamma_{1} a_{2}+\xi \gamma_{2} a_{5}+\left(\delta+\eta_{h}\right)<1
$$

Proof We initiate with $\Omega_{1}$. For two functions $S_{h}$ and $S_{h 1}$, we have

$$
\begin{aligned}
& \left\|\Omega_{1}\left(t, S_{h}\right)-\Omega_{1}\left(t, S_{h 1}\right)\right\| \\
& \quad=\left\|\left\{S_{h}(t)-S_{h 1}(t)\right\}\left(\omega \gamma_{1} I_{h}(t)+\xi \gamma_{2} I_{m}(t)\right)-\left\{S_{h}(t)-S_{h 1}(t)\right\}\left(\delta+\eta_{h}\right)\right\| .
\end{aligned}
$$

On applying the properties of norm on Eq. (11), it yields

$$
\begin{aligned}
\left\|\Omega_{1}\left(t, S_{h}\right)-\Omega_{1}\left(t, S_{h 1}\right)\right\| \leq & \left\|\left\{S_{h}(t)-S_{h 1}(t)\right\}\left(\omega \gamma_{1} I_{h}(t)+\xi \gamma_{2} I_{m}(t)\right)\right\| \\
& +\left\|\left\{S_{h}(t)-S_{h 1}(t)\right\}\left(\delta+\eta_{h}\right)\right\| \\
\leq & \left\{\omega \gamma_{1}\left\|I_{h}(t)\right\|+\xi \gamma_{2}\left\|I_{m}(t)\right\|+\left(\delta+\eta_{h}\right)\right\}\left\|S_{h}(t)-S_{h 1}(t)\right\| \\
\leq & \left\{\omega \gamma_{1} a_{2}+\xi \gamma_{2} a_{5}+\left(\delta+\eta_{h}\right)\right\}\left\|S_{h}(t)-S_{h 1}(t)\right\| \\
\leq & b_{1}\left\|S_{h}(t)-S_{h 1}(t)\right\| .
\end{aligned}
$$

Taking $b_{1}=\omega \gamma_{1} a_{2}+\xi \gamma_{2} a_{5}+\left(\delta+\eta_{h}\right)$, where $\left\|S_{h}(t)\right\| \leq a_{1},\left\|I_{h}(t)\right\| \leq a_{2},\left\|R_{h}(t)\right\| \leq a_{3}$, $\left\|S_{m}(t)\right\| \leq a_{4}$, and $\left\|I_{m}(t)\right\| \leq a_{5}$ are bounded functions, we get

$$
\left\|\Omega_{1}\left(t, S_{h}\right)-\Omega_{1}\left(t, S_{h 1}\right)\right\| \leq b_{1}\left\|S_{h}(t)-S_{h 1}(t)\right\|
$$

Thus the Lipschitz condition is satisfied for $\Omega_{1}$. Furthermore, if

$$
0 \leq \omega \gamma_{1} a_{2}+\xi \gamma_{2} a_{5}+\left(\delta+\eta_{h}\right)<1, \quad \text { then it is also a contraction. }
$$

Similarly, we can prove that the kernels $\Omega_{2}\left(t, I_{h}\right), \Omega_{3}\left(t, R_{h}\right), \Omega_{4}\left(t, S_{m}\right)$, and $\Omega_{5}\left(t, I_{m}\right)$ satisfy the Lipschitz conditions

$$
\begin{aligned}
& \left\|\Omega_{2}\left(t, I_{h}\right)-\Omega_{2}\left(t, I_{h 1}\right)\right\| \leq b_{2}\left\|I_{h}(t)-I_{h 1}(t)\right\|, \\
& \left\|\Omega_{3}\left(t, R_{h}\right)-\Omega_{3}\left(t, R_{h 1}\right)\right\| \leq b_{3}\left\|R_{h}(t)-R_{h 1}(t)\right\|, \\
& \left\|\Omega_{4}\left(t, S_{m}\right)-\Omega_{4}\left(t, S_{m 1}\right)\right\| \leq b_{4}\left\|S_{m}(t)-S_{m 1}(t)\right\|, \\
& \left\|\Omega_{5}\left(t, I_{m}\right)-\Omega_{5}\left(t, I_{m 1}\right)\right\| \leq b_{5}\left\|I_{m}(t)-I_{m 1}(t)\right\| .
\end{aligned}
$$


On using the notations of the earlier stated kernels, Eq. (9) reduces to the system

$$
\begin{aligned}
& S_{h}(t)=S_{h}(0)+\frac{2(1-\lambda)}{(2-\lambda) M(\lambda)} \Omega_{1}\left(t, S_{h}\right)+\frac{2 \lambda}{(2-\lambda) M(\lambda)} \int_{0}^{t} \Omega_{1}\left(\varsigma, S_{h}\right) d \varsigma, \\
& I_{h}(t)=I_{h}(0)+\frac{2(1-\lambda)}{(2-\lambda) M(\lambda)} \Omega_{2}\left(t, I_{h}\right)+\frac{2 \lambda}{(2-\lambda) M(\lambda)} \int_{0}^{t} \Omega_{2}\left(\varsigma, I_{h}\right) d \varsigma, \\
& R_{h}(t)=R_{h}(0)+\frac{2(1-\lambda)}{(2-\lambda) M(\lambda)} \Omega_{3}\left(t, R_{h}\right)+\frac{2 \lambda}{(2-\lambda) M(\lambda)} \int_{0}^{t} \Omega_{3}\left(\varsigma, R_{h}\right) d \varsigma, \\
& S_{m}(t)=S_{m}(0)+\frac{2(1-\lambda)}{(2-\lambda) M(\lambda)} \Omega_{4}\left(t, S_{m}\right)+\frac{2 \lambda}{(2-\lambda) M(\lambda)} \int_{0}^{t} \Omega_{4}\left(\varsigma, S_{m}\right) d \varsigma, \\
& I_{m}(t)=I_{m}(0)+\frac{2(1-\lambda)}{(2-\lambda) M(\lambda)} \Omega_{5}\left(t, I_{m}\right)+\frac{2 \lambda}{(2-\lambda) M(\lambda)} \int_{0}^{t} \Omega_{5}\left(\varsigma, I_{m}\right) d \varsigma .
\end{aligned}
$$

Next, we construct the following recursive formulas:

$$
\begin{aligned}
& S_{h n}(t)=\frac{2(1-\lambda)}{(2-\lambda) M(\lambda)} \Omega_{1}\left(t, S_{h(n-1)}\right)+\frac{2 \lambda}{(2-\lambda) M(\lambda)} \int_{0}^{t}\left(\Omega_{1}\left(\varsigma, S_{h(n-1)}\right)\right) d \varsigma, \\
& I_{h n}(t)=\frac{2(1-\lambda)}{(2-\lambda) M(\lambda)} \Omega_{2}\left(t, I_{h(n-1)}\right)+\frac{2 \lambda}{(2-\lambda) M(\lambda)} \int_{0}^{t}\left(\Omega_{2}\left(\varsigma, I_{h(n-1)}\right)\right) d \varsigma, \\
& R_{h n}(t)=\frac{2(1-\lambda)}{(2-\lambda) M(\lambda)} \Omega_{3}\left(t, R_{h(n-1)}\right)+\frac{2 \lambda}{(2-\lambda) M(\lambda)} \int_{0}^{t}\left(\Omega_{3}\left(\varsigma, R_{h(n-1)}\right)\right) d \varsigma, \\
& S_{m n}(t)=\frac{2(1-\lambda)}{(2-\lambda) M(\lambda)} \Omega_{4}\left(t, S_{m(n-1)}\right)+\frac{2 \lambda}{(2-\lambda) M(\lambda)} \int_{0}^{t}\left(\Omega_{4}\left(\varsigma, S_{m(n-1)}\right)\right) d \varsigma, \\
& I_{m n}(t)=\frac{2(1-\lambda)}{(2-\lambda) M(\lambda)} \Omega_{5}\left(t, I_{m(n-1)}\right)+\frac{2 \lambda}{(2-\lambda) M(\lambda)} \int_{0}^{t}\left(\Omega_{5}\left(\varsigma, I_{m(n-1)}\right)\right) d \varsigma,
\end{aligned}
$$

along with the initial conditions

$$
\begin{array}{ll}
S_{h 0}=S_{h}(0), & I_{h 0}=I_{h}(0), \quad R_{h 0}=R_{h}(0), \\
S_{m 0}=S_{m}(0), & I_{m 0}=I_{m}(0) .
\end{array}
$$

We express the difference between the succession terms as

$$
\begin{aligned}
\varpi_{1 n}(t)= & S_{h n}(t)-S_{h(n-1)}(t) \\
= & \frac{2(1-\lambda)}{(2-\lambda) M(\lambda)}\left(\Omega_{1}\left(t, S_{h(n-1)}\right)-\Omega_{1}\left(t, S_{h(n-2)}\right)\right) \\
& +\frac{2 \lambda}{(2-\lambda) M(\lambda)} \int_{0}^{t}\left(\Omega_{1}\left(\varsigma, S_{h(n-1)}\right)-\Omega_{1}\left(\varsigma, S_{h(n-2)}\right)\right) d \varsigma, \\
\varpi_{2 n}(t)= & I_{h n}(t)-I_{h(n-1)}(t) \\
= & \frac{2(1-\lambda)}{(2-\lambda) M(\lambda)}\left(\Omega_{2}\left(t, I_{h(n-1)}\right)-\Omega_{2}\left(t, I_{h(n-2)}\right)\right) \\
& +\frac{2 \lambda}{(2-\lambda) M(\lambda)} \int_{0}^{t}\left(\Omega_{2}\left(\varsigma, I_{h(n-1)}\right)-\Omega_{2}\left(\varsigma, I_{h(n-2)}\right)\right) d \varsigma,
\end{aligned}
$$




$$
\begin{aligned}
\varpi_{3 n}(t)= & R_{h n}(t)-R_{h(n-1)}(t) \\
= & \frac{2(1-\lambda)}{(2-\lambda) M(\lambda)}\left(\Omega_{3}\left(t, R_{h(n-1)}\right)-\Omega_{3}\left(t, R_{h(n-2)}\right)\right) \\
& +\frac{2 \lambda}{(2-\lambda) M(\lambda)} \int_{0}^{t}\left(\Omega_{3}\left(\varsigma, R_{h(n-1)}\right)-\Omega_{3}\left(\varsigma, R_{h(n-2)}\right)\right) d \zeta, \\
\varpi_{4 n}(t)= & S_{m n}(t)-S_{m(n-1)}(t) \\
= & \frac{2(1-\lambda)}{(2-\lambda) M(\lambda)}\left(\Omega_{4}\left(t, S_{m(n-1)}\right)-\Omega_{4}\left(t, S_{m(n-2)}\right)\right) \\
& +\frac{2 \lambda}{(2-\lambda) M(\lambda)} \int_{0}^{t}\left(\Omega_{4}\left(\zeta, S_{h(n-1)}\right)-\Omega_{4}\left(\zeta, S_{m(n-2)}\right)\right) d \zeta, \\
\varpi_{5 n}(t)= & I_{m n}(t)-I_{m(n-1)}(t) \\
= & \frac{2(1-\lambda)}{(2-\lambda) M(\lambda)}\left(\Omega_{5}\left(t, I_{m(n-1)}\right)-\Omega_{5}\left(t, I_{m(n-2)}\right)\right) \\
& +\frac{2 \lambda}{(2-\lambda) M(\lambda)} \int_{0}^{t}\left(\Omega_{5}\left(\zeta, I_{h(n-1)}\right)-\Omega_{5}\left(\zeta, I_{m(n-2)}\right)\right) d \zeta .
\end{aligned}
$$

It is worth observing that

$$
\begin{array}{ll}
S_{h n}(t)=\sum_{i=0}^{n} \varpi_{1 i}(t), & I_{h n}(t)=\sum_{i=0}^{n} \varpi_{2 i}(t), \quad R_{h n}(t)=\sum_{i=0}^{n} \varpi_{3 i}(t), \\
S_{m n}(t)=\sum_{i=0}^{n} \varpi_{4 i}(t), & I_{m n}(t)=\sum_{i=0}^{n} \varpi_{5 i}(t) .
\end{array}
$$

Now we easily obtain the following result:

$$
\begin{aligned}
\left\|\varpi_{1 n}(t)\right\| & =\left\|S_{h n}(t)-S_{h(n-1)}(t)\right\| \\
& =\| \begin{array}{l}
\frac{2(1-\lambda)}{(2-\lambda) M(\lambda)}\left(\Omega_{1}\left(t, S_{h(n-1)}\right)-\Omega_{1}\left(t, S_{h(n-2)}\right)\right) \\
+\frac{2 \lambda}{(2-\lambda) M(\lambda)} \int_{0}^{t}\left(\Omega_{1}\left(\varsigma, S_{h(n-1)}\right)-\Omega_{1}\left(\varsigma, S_{h(n-2)}\right)\right) d \varsigma
\end{array}
\end{aligned} .
$$

Applying the triangle inequality to Eq. (20), we get

$$
\begin{aligned}
\left\|S_{h n}(t)-S_{h(n-1)}(t)\right\| \leq & \frac{2(1-\lambda)}{(2-\lambda) M(\lambda)}\left(\Omega_{1}\left(t, S_{h(n-1)}\right)-\Omega_{1}\left(t, S_{h(n-2)}\right)\right. \\
& +\frac{2 \lambda}{(2-\lambda) M(\lambda)}\left\|\int_{0}^{t}\left(\Omega_{1}\left(\varsigma, S_{h(n-1)}\right)-\Omega_{1}\left(\varsigma, S_{h(n-2)}\right)\right) d \varsigma\right\| .
\end{aligned}
$$

It is already proved that the kernels satisfy the Lipschitz condition, so Eq. (21) gives

$$
\begin{aligned}
\left\|S_{h n}(t)-S_{h(n-1)}(t)\right\| \leq & \frac{2(1-\lambda)}{(2-\lambda) M(\lambda)} b_{1}\left\|S_{h(n-1)}-S_{h(n-2)}\right\| \\
& +\frac{2 \lambda}{(2-\lambda) M(\lambda)} b_{1} \int_{0}^{t}\left\|S_{h(n-1)}-S_{h(n-2)}\right\| d \zeta .
\end{aligned}
$$


Consequently, we arrive at the subsequent result

$$
\left\|\varpi_{1 n}(t)\right\| \leq \frac{2(1-\lambda)}{(2-\lambda) M(\lambda)} b_{1}\left\|\varpi_{1(n-1)}(t)\right\|+\frac{2 \lambda}{(2-\lambda) M(\lambda)} b_{1} \int_{0}^{t}\left\|\varpi_{1(n-1)}(\varsigma)\right\| d \varsigma .
$$

Using the same process, we derive the following results:

$$
\begin{aligned}
\left\|\varpi_{2 n}(t)\right\| & \leq \frac{2(1-\lambda)}{(2-\lambda) M(\lambda)} b_{2}\left\|\varpi_{2(n-1)}(t)\right\|+\frac{2 \lambda}{(2-\lambda) M(\lambda)} b_{2} \int_{0}^{t}\left\|\varpi_{2(n-1)}(\varsigma)\right\| d \varsigma, \\
\left\|\varpi_{3 n}(t)\right\| & \leq \frac{2(1-\lambda)}{(2-\lambda) M(\lambda)} b_{3}\left\|\varpi_{3(n-1)}(t)\right\|+\frac{2 \lambda}{(2-\lambda) M(\lambda)} b_{3} \int_{0}^{t}\left\|\varpi_{3(n-1)}(\varsigma)\right\| d \varsigma, \\
\left\|\varpi_{4 n}(t)\right\| & \leq \frac{2(1-\lambda)}{(2-\lambda) M(\lambda)} b_{4}\left\|\varpi_{4(n-1)}(t)\right\|+\frac{2 \lambda}{(2-\lambda) M(\lambda)} b_{4} \int_{0}^{t}\left\|\varpi_{4(n-1)}(\varsigma)\right\| d \varsigma, \\
\left\|\varpi_{5 n}(t)\right\| & \leq \frac{2(1-\lambda)}{(2-\lambda) M(\lambda)} b_{5}\left\|\varpi_{5(n-1)}(t)\right\|+\frac{2 \lambda}{(2-\lambda) M(\lambda)} b_{5} \int_{0}^{t}\left\|\varpi_{5(n-1)}(\varsigma)\right\| d \zeta .
\end{aligned}
$$

Taking (23) and (24) into account, we obtain the existence of the solution of the considered model.

Theorem 2 The SIRS-SI malaria model involving the CF fractional operator expressed in $E q$. (5) has a solution if there exists $t_{0}$ such that

$$
\frac{2(1-\lambda)}{(2-\lambda) M(\lambda)} b_{1}+\frac{2 \lambda}{(2-\lambda) M(\lambda)} b_{1} t_{0}<1
$$

Proof As we know, the functions $S_{h}(t), I_{h}(t), R_{h}(t), S_{m}(t)$, and $I_{m}(t)$ are bounded. Using the results presented in Eqs. (23)-(24) and utilizing the recursive algorithm, we get

$$
\begin{aligned}
& \left\|\varpi_{1 n}(t)\right\| \leq\left\|S_{h n}(0)\right\|\left[\frac{2(1-\lambda)}{(2-\lambda) M(\lambda)} b_{1}+\frac{2 \lambda}{(2-\lambda) M(\lambda)} b_{1} t\right]^{n}, \\
& \left\|\varpi_{2 n}(t)\right\| \leq\left\|I_{h n}(0)\right\|\left[\frac{2(1-\lambda)}{(2-\lambda) M(\lambda)} b_{2}+\frac{2 \lambda}{(2-\lambda) M(\lambda)} b_{2} t\right]^{n}, \\
& \left\|\varpi_{3 n}(t)\right\| \leq\left\|R_{h n}(0)\right\|\left[\frac{2(1-\lambda)}{(2-\lambda) M(\lambda)} b_{3}+\frac{2 \lambda}{(2-\lambda) M(\lambda)} b_{3} t\right]^{n}, \\
& \left\|\varpi_{4 n}(t)\right\| \leq\left\|S_{m n}(0)\right\|\left[\frac{2(1-\lambda)}{(2-\lambda) M(\lambda)} b_{4}+\frac{2 \lambda}{(2-\lambda) M(\lambda)} b_{4} t\right]^{n}, \\
& \left\|\varpi_{5 n}(t)\right\| \leq\left\|I_{m n}(0)\right\|\left[\frac{2(1-\lambda)}{(2-\lambda) M(\lambda)} b_{5}+\frac{2 \lambda}{(2-\lambda) M(\lambda)} b_{5} t\right]^{n} .
\end{aligned}
$$

Hence the solution of the considered model exists and is continuous.

Now, to show that Eq. (15) is a solution of the model (5), we take

$$
\begin{aligned}
& S_{h}(t)-S_{h}(0)=S_{n h}(t)-A_{n}(t), \\
& I_{h}(t)-I_{h}(0)=I_{h n}(t)-B_{n}(t), \\
& R_{h}(t)-R_{h}(0)=R_{h n}(t)-C_{n}(t), \\
& S_{m}(t)-S_{m}(0)=S_{m n}(t)-D_{n}(t), \\
& I_{m}(t)-I_{m}(0)=I_{m n}(t)-E_{n}(t) .
\end{aligned}
$$


Thus we have

$$
\begin{aligned}
\left\|A_{n}(t)\right\|= & \| \frac{2(1-\lambda)}{(2-\lambda) M(\lambda)}\left(\Omega_{1}\left(t, S_{h}\right)-\Omega_{1}\left(t, S_{h(n-1)}\right)\right) \\
& +\frac{2 \lambda}{(2-\lambda) M(\lambda)} \int_{0}^{t}\left(\Omega_{1}\left(\varsigma, S_{h}\right)-\Omega_{1}\left(\varsigma, S_{h(n-1)}\right)\right) d \varsigma \| \\
\leq & \left.\frac{2(1-\lambda)}{(2-\lambda) M(\lambda)} \|\left(\Omega_{1}\left(t, S_{h}\right)-\Omega_{1}\left(t, S_{h(n-1)}\right)\right)\right) \| \\
& +\frac{2 \lambda}{(2-\lambda) M(\lambda)} \int_{0}^{t}\left\|\left(\Omega_{1}\left(\varsigma, S_{h}\right)-\Omega_{1}\left(\varsigma, S_{h(n-1)}\right)\right)\right\| d \varsigma \\
\leq & \frac{2(1-\lambda)}{(2-\lambda) M(\lambda)} b_{1}\left\|S_{h}-S_{h(n-1)}\right\|+\frac{2 \lambda}{(2-\lambda) M(\lambda)} b_{1}\left\|S_{h}-S_{h(n-1)}\right\| t .
\end{aligned}
$$

Using this process recursively, we get

$$
\left\|A_{n}(t)\right\| \leq\left(\frac{2(1-\lambda)}{(2-\lambda) M(\lambda)}+\frac{2 \lambda}{(2-\lambda) M(\lambda)} t\right)^{n+1} b_{1}^{n+1} a_{1}
$$

Then at $t_{0}$, we have

$$
\left\|A_{n}(t)\right\| \leq\left(\frac{2(1-\lambda)}{(2-\lambda) M(\lambda)}+\frac{2 \lambda}{(2-\lambda) M(\lambda)} t_{0}\right)^{n+1} b_{1}^{n+1} a_{1} .
$$

Taking the limit on Eq. (29) as $n$ tends to infinity gives

$$
\left\|A_{n}(t)\right\| \rightarrow 0
$$

Similarly, we get

$$
\left\|B_{n}(t)\right\| \rightarrow 0, \quad\left\|C_{n}(t)\right\| \rightarrow 0, \quad\left\|D_{n}(t)\right\| \rightarrow 0, \quad \text { and } \quad\left\|E_{n}(t)\right\| \rightarrow 0 .
$$

This completes the proof of the existence theorem.

Next, we prove the uniqueness of a solution of the fractional SIRS-SI malaria model (5).

Let us assume that there exists another system of solutions of SIRS-SI malaria model $(5), S_{h}^{*}(t), I_{h}^{*}(t), R_{h}^{*}(t), S_{m}^{*}(t)$, and $I_{m}^{*}(t)$. Then

$$
\begin{aligned}
S_{h}(t)-S_{h}^{*}(t)= & \frac{2(1-\lambda)}{(2-\lambda) M(\lambda)}\left(\Omega_{1}\left(t, S_{h}\right)-\Omega_{1}\left(t, S_{h}^{*}\right)\right) \\
& +\frac{2 \lambda}{(2-\lambda) M(\lambda)} \int_{0}^{t}\left(\Omega_{1}(\varsigma, S)-\Omega_{1}\left(\varsigma, S_{h}^{*}\right)\right) d \varsigma .
\end{aligned}
$$

Taking the norms gives

$$
\begin{aligned}
\left\|S_{h}(t)-S_{h}^{*}(t)\right\| \leq & \frac{2(1-\lambda)}{(2-\lambda) M(\lambda)}\left\|\Omega_{1}\left(t, S_{h}\right)-\Omega_{1}\left(t, S_{h}^{*}\right)\right\| \\
& +\frac{2 \lambda}{(2-\lambda) M(\lambda)} \int_{0}^{t}\left\|\left(\Omega_{1}(\varsigma, S)-\Omega_{1}\left(\varsigma, S_{h}^{*}\right)\right)\right\| d \varsigma .
\end{aligned}
$$


Employing the results presented in (13) and (14), we get

$$
\begin{aligned}
\left\|S(t)-S_{h}^{*}(t)\right\| \leq & \frac{2(1-\lambda)}{(2-\lambda) M(\lambda)} b_{1}\left\|S_{h}(t)-S_{h}^{*}(t)\right\| \\
& +\frac{2 \lambda}{(2-\lambda) M(\lambda)} b_{1} t\left\|S_{h}(t)-S_{h}^{*}(t)\right\|,
\end{aligned}
$$

which gives

$$
\left\|S_{h}(t)-S_{h}^{*}(t)\right\|\left(1-\frac{2(1-\lambda)}{(2-\lambda) M(\lambda)} b_{1}-\frac{2 \lambda}{(2-\lambda) M(\lambda)} b_{1} t\right) \leq 0 .
$$

Theorem 3 The fractional SIRS-SI malaria model (5) has a unique solution if

$$
\left(1-\frac{2(1-\lambda)}{(2-\lambda) M(\lambda)} \gamma_{1}-\frac{2 \lambda}{(2-\lambda) M(\lambda)} \gamma_{1} t\right)>0
$$

Proof From Eq. (33) we have

$$
\left\|S_{h}(t)-S_{h}^{*}(t)\right\|\left(1-\frac{2(1-\lambda)}{(2-\lambda) M(\lambda)} b_{1}-\frac{2 \lambda}{(2-\lambda) M(\lambda)} b_{1} t\right) \leq 0 .
$$

Using Eq. (34) and properties of a norm in Eq. (35) gives

$$
\left\|S_{h}(t)-S_{h}^{*}(t)\right\|=0
$$

Thus we can see that

$$
S_{h}(t)=S_{h}^{*}(t) .
$$

Using a similar procedure, we easily prove that

$$
I_{h}=I_{h}^{*}, \quad R_{h}=R_{h}^{*}, \quad S_{m}=S_{m}^{*}, \quad I_{m}=I_{m}^{*} .
$$

Thus the fractional SIRS-SI malaria model (5) has a unique solution.

\section{HATM for fractional SIRS-SI malaria model}

In this section, we simulate the numerical results for the fractional SIRS-SI malaria model by using HATM. Firstly, we apply the Laplace transform to fractional SIRS-SI malaria model (5), which yields

$$
\begin{aligned}
& \frac{s L\left[S_{h}\right]-S_{h}(0)}{s+\lambda(1-s)}=L\left[\alpha_{h}+\beta R_{h}-\left(\omega \gamma_{1} I_{h}+\xi \gamma_{2} I_{m}\right) S_{h}-\left(\delta+\eta_{h}\right) S_{h}\right], \\
& \frac{s L\left[I_{h}\right]-I_{h}(0)}{s+\lambda(1-s)}=L\left[\mu I_{h}+\left(\omega \gamma_{1} I_{h}+\xi \gamma_{2} I_{m}\right) S_{h}-\left(\eta_{h}+\varepsilon+c \nu\right) I_{h}\right], \\
& \frac{s L\left[R_{h}\right]-R_{h}(0)}{s+\lambda(1-s)}=L\left[c v I_{h}-\left(\eta_{h}+\beta\right) R_{h}+\delta S_{h}\right],
\end{aligned}
$$




$$
\begin{aligned}
& \frac{s L\left[S_{m}\right]-S_{m}(0)}{s+\lambda(1-s)}=L\left[\alpha_{m}-\left(e \gamma_{3} I_{h}+\eta_{m}+\sigma\right) S_{m}\right], \\
& \frac{s L\left[I_{m}\right]-I_{m}(0)}{s+\lambda(1-s)}=L\left[e \gamma_{3} I_{h} S_{m}-\left(\eta_{m}+\sigma\right) I_{m}\right] .
\end{aligned}
$$

By simplification this gives

$$
\begin{aligned}
& L\left[S_{h}\right]-\frac{c_{1}}{s}-\frac{[s+\lambda(1-s)] \alpha_{h}}{s^{2}} \\
& \quad-\frac{s+\lambda(1-s)}{s} L\left[\beta R_{h}-\left(\omega \gamma_{1} I_{h}+\xi \gamma_{2} I_{m}\right) S_{h}-\left(\delta+\eta_{h}\right) S_{h}\right]=0 \\
& L\left[I_{h}\right]-\frac{c_{2}}{s}-\frac{s+\lambda(1-s)}{s} L\left[\mu I_{h}+\left(\omega \gamma_{1} I_{h}+\xi \gamma_{2} I_{m}\right) S_{h}-\left(\eta_{h}+\varepsilon+c \nu\right) I_{h}\right]=0 \\
& L\left[R_{h}\right]-\frac{c_{3}}{s}-\frac{s+\lambda(1-s)}{s} L\left[c \nu I_{h}-\left(\eta_{h}+\beta\right) R_{h}+\delta S_{h}\right]=0 \\
& L\left[S_{m}\right]-\frac{c_{4}}{s}-\frac{[s+\lambda(1-s)] \alpha_{m}}{s^{2}}-\frac{s+\lambda(1-s)}{s} L\left[-\left(e \gamma_{3} I_{h}+\eta_{m}+\sigma\right) S_{m}\right]=0 \\
& L\left[I_{m}\right]-\frac{c_{5}}{s}-\frac{s+\lambda(1-s)}{s} L\left[e \gamma_{3} I_{h} S_{m}-\left(\eta_{m}+\sigma\right) I_{m}\right]=0
\end{aligned}
$$

We present the nonlinear operators as

$$
\begin{aligned}
N_{1}\left[\varphi_{1}(t ; z)\right]= & L\left[\varphi_{1}(t ; z)\right]-\frac{c_{1}}{s}-\frac{[s+\lambda(1-s)] \alpha_{h}}{s^{2}}-\frac{s+\lambda(1-s)}{s} L\left[\beta \varphi_{3}(t ; z)\right. \\
& \left.-\left(\omega \gamma_{1} \varphi_{2}(t ; z)+\xi \gamma_{2} \varphi_{5}(t ; z)\right) \varphi_{1}(t ; z)-\left(\delta+\eta_{h}\right) \varphi_{1}(t ; z)\right]=0, \\
N_{2}\left[\varphi_{2}(t ; z)\right]= & L\left[\varphi_{2}(t ; z)\right]-\frac{c_{2}}{s}-\frac{s+\lambda(1-s)}{s} L\left[\mu \varphi_{2}(t ; z)\right. \\
& \left.+\left(\omega \gamma_{1} \varphi_{2}(t ; z)+\xi \gamma_{2} \varphi_{4}(t ; z)\right) \varphi_{1}(t ; z)-\left(\eta_{h}+\varepsilon+c \nu\right) \varphi_{2}(t ; z)\right]=0, \\
N_{3}\left[\varphi_{3}(t ; z)\right]= & L\left[\varphi_{3}(t ; z)\right]-\frac{c_{3}}{s}-\frac{s+\lambda(1-s)}{s} L\left[c v \varphi_{2}(t ; z)\right. \\
& \left.-\left(\eta_{h}+\beta\right) \varphi_{3}(t ; z)+\delta \varphi_{1}(t ; z)\right]=0 \\
N_{4}\left[\varphi_{4}(t ; z)\right]= & L\left[\varphi_{4}(t ; z)\right]-\frac{c_{4}}{s}-\frac{[s+\lambda(1-s)] \alpha_{m}}{s^{2}} \\
& -\frac{s+\lambda(1-s)}{s} L\left[-\left(e \gamma_{3} \varphi_{2}(t ; z)+\eta_{m}+\sigma\right) \varphi_{4}(t ; z)\right] \\
& \left.-\left(\eta_{m}+\sigma\right) \varphi_{5}(t ; z)\right]=0, \\
N_{5}\left[\varphi_{5}(t ; z)\right]= & L\left[\varphi_{5}(t ; z)\right]-\frac{c_{5}}{s}-\frac{s+\lambda(1-s)}{s} L\left[e \gamma_{3} \varphi_{2}(t ; z) \varphi_{4}(t ; z)\right. \\
&
\end{aligned}
$$

and thus we have

$$
\begin{aligned}
\mathfrak{R}_{1, k}\left(\vec{S}_{h(k-1)}\right)= & L\left[S_{h(k-1)}\right]-\left(\frac{c_{1}}{s}+\frac{[s+\lambda(1-s)] \alpha_{h}}{s^{2}}\right)\left(1-\chi_{k}\right) \\
& -\frac{s+\lambda(1-s)}{s} L\left[\beta R_{h(k-1)}-\omega \gamma_{1}\left(\sum_{r=0}^{k-1} I_{h r} S_{h(k-1-r)}\right)\right. \\
& \left.-\xi \gamma_{2}\left(\sum_{r=0}^{k-1} I_{m r} S_{h(k-1-r)}\right)-\left(\delta+\eta_{h}\right) S_{h(k-1)}\right]
\end{aligned}
$$




$$
\begin{aligned}
& \Re_{2, k}\left(\vec{I}_{h(k-1)}\right)=L\left[I_{h(k-1)}\right]-\frac{c_{2}}{s}\left(1-\chi_{k}\right) \\
& -\frac{s+\lambda(1-s)}{s} L\left[\mu I_{h(k-1)}+\omega \gamma_{1}\left(\sum_{r=0}^{k-1} I_{h r} S_{h(k-1-r)}\right)\right. \\
& \left.+\xi \gamma_{2}\left(\sum_{r=0}^{k-1} S_{m r} S_{h(k-1-r)}\right)-\left(\eta_{h}+\varepsilon+c \nu\right) I_{h(k-1)}\right] \\
& \Re_{3, k}\left(\vec{R}_{h(k-1)}\right)=L\left[R_{h(k-1)}\right]-\frac{c_{3}}{s}\left(1-\chi_{k}\right) \\
& -\frac{s+\lambda(1-s)}{s} L\left[c \nu I_{h(k-1)}-\left(\eta_{h}+\beta\right) R_{h(k-1)}+\delta S_{h(k-1)}\right], \\
& \mathfrak{R}_{4, k}\left(\vec{S}_{m(k-1)}\right)=L\left[S_{m(k-1)}\right]-\left(\frac{c_{4}}{s}+\frac{[s+\lambda(1-s)] \alpha_{m}}{s^{2}}\right)\left(1-\chi_{k}\right) \\
& -\frac{s+\lambda(1-s)}{s} L\left[-e \gamma_{3}\left(\sum_{r=0}^{k-1} I_{h r} S_{m(k-1-r)}\right)-\left(\eta_{m}+\sigma\right) S_{m(k-1)}\right], \\
& \Re_{5, k}\left(\vec{I}_{m(k-1)}\right)=L\left[I_{m(k-1)}\right]-\frac{c_{5}}{s}\left(1-\chi_{k}\right) \\
& -\frac{s+\lambda(1-s)}{s} L\left[e \gamma_{3}\left(\sum_{r=0}^{k-1} I_{h r} S_{m(k-1-r)}\right)-\left(\eta_{m}+\sigma\right) I_{m(k-1)}\right] .
\end{aligned}
$$

Further, the deformation equations of $k$ th order are expressed as

$$
\begin{aligned}
& L\left[S_{h k}(t)-\chi_{k} S_{h(k-1)}(t)\right]=\hbar \Re_{1, k}\left(\vec{S}_{h(k-1)}\right), \\
& L\left[I_{h k}(t)-\chi_{k} I_{h(k-1)}(t)\right]=\hbar \Re_{2, k}\left(\vec{I}_{h(k-1)}\right), \\
& L\left[R_{h k}(t)-\chi_{k} R_{h(k-1)}(t)\right]=\hbar \Re_{3, k}\left(\vec{R}_{h(k-1)}\right), \\
& L\left[S_{m k}(t)-\chi_{k} S_{m(k-1)}(t)\right]=\hbar \Re_{4, k}\left(\vec{S}_{m(k-1)}\right), \\
& L\left[I_{m k}(t)-\chi_{k} I_{m(k-1)}(t)\right]=\hbar \Re_{5, k}\left(\vec{I}_{m(k-1)}\right) .
\end{aligned}
$$

Applying the inverse Laplace transform to Eq. (42) yields

$$
\begin{aligned}
& S_{h k}(t)=\chi_{k} S_{h(k-1)}(t)+\hbar L^{-1}\left[\Re_{1, k}\left(\vec{S}_{h(k-1)}\right)\right], \\
& I_{h k}(t)=\chi_{k} I_{h(k-1)}(t)+\hbar L^{-1}\left[\Re_{2, k}\left(\vec{I}_{h(k-1)}\right)\right] \\
& R_{h k}(t)=\chi_{k} R_{h(k-1)}(t)+\hbar L^{-1}\left[\Re_{3, k}\left(\vec{R}_{h(k-1)}\right)\right] \\
& S_{m k}(t)=\chi_{k} S_{m(k-1)}(t)+\hbar L^{-1}\left[\Re_{4, k}\left(\vec{S}_{m(k-1)}\right)\right] \\
& I_{m k}(t)=\chi_{k} I_{m(k-1)}(t)+\hbar L^{-1}\left[\Re_{5, k}\left(\vec{I}_{m(k-1)}\right)\right] .
\end{aligned}
$$

Taking the initial guess $S_{h 0}(t)=c_{1}+\{1+\lambda(t-1)\} \alpha_{h}, I_{h 0}(t)=c_{2}, R_{h 0}(t)=c_{3}, S_{m 0}(t)=c_{4}+\{1+$ $\lambda(t-1)\} \alpha_{m}, I_{m 0}(t)=c_{5}$ and solving Eq. (43) for $k=1,2,3, \ldots$, we get the values of $S_{h k}(t)$, $I_{h k}(t), R_{h k}(t), S_{m k}(t)$, and $I_{m k}(t)$ for $k \geq 1$.

So, the solution of fractional SIRS-SI malaria model (5) is given as

$$
\begin{aligned}
& S_{h}(t)=S_{h 0}+S_{h 1}+S_{h 2}+\cdots, \\
& I_{h}(t)=I_{h 0}+I_{h 1}+I_{h 2}+\cdots, \\
& R_{h}(t)=R_{h 0}+R_{h 1}+R_{h 2}+\cdots,
\end{aligned}
$$




$$
\begin{aligned}
& S_{m}(t)=S_{m 0}+S_{m 1}+S_{m 2}+\cdots, \\
& I_{m}(t)=I_{m 0}+I_{m 1}+I_{m 2}+\cdots .
\end{aligned}
$$

\section{Numerical results and discussions}

This part is devoted to numerical simulation for the fractional SIRS-SI malaria model. The numerical results for the model (5) are computed by using the HATM and Padé approximation. To compute numerical results, we have taken the values of various parameters from distinct reliable sources $[1,2,5]$. The numerical simulation is performed fort $\eta_{h}=0.0004, \eta_{h}=0.04, \varepsilon=0.05, \beta=1 / 730, \sigma=1 / 730, \alpha_{h}=0.027, \alpha_{m}=0.13, \omega=0.038$, $\xi=0.13, \gamma_{1}=0.02, \gamma_{2}=0.010, \gamma_{3}=0.072, c=0.611, e=0.022, \mu=0.005, \sigma \in[0,1]$, $\delta \in[0,1]$, and $v \in[0.01,1]$. The initial conditions are taken as $S_{h}(0)=c_{1}=40, I_{h}(0)=c_{2}=2$, $R_{h}(0)=c_{3}=0, S_{m}(0)=c_{4}=500$, and $I_{m}(0)=c_{5}=10$. Figure 1 depicts the influence of order of the $\mathrm{CF}$ fractional operator on different groups of human population, that is, susceptible humans, infected humans, and recovered humans. Figure 2 demonstrates the influence of order of the CF fractional derivative on different groups of mosquito population, that is, susceptible mosquitoes and infected mosquitoes. Figure 3 shows the influence of antimalarial drugs on different classes of human population. Figure 4 displays the impact of
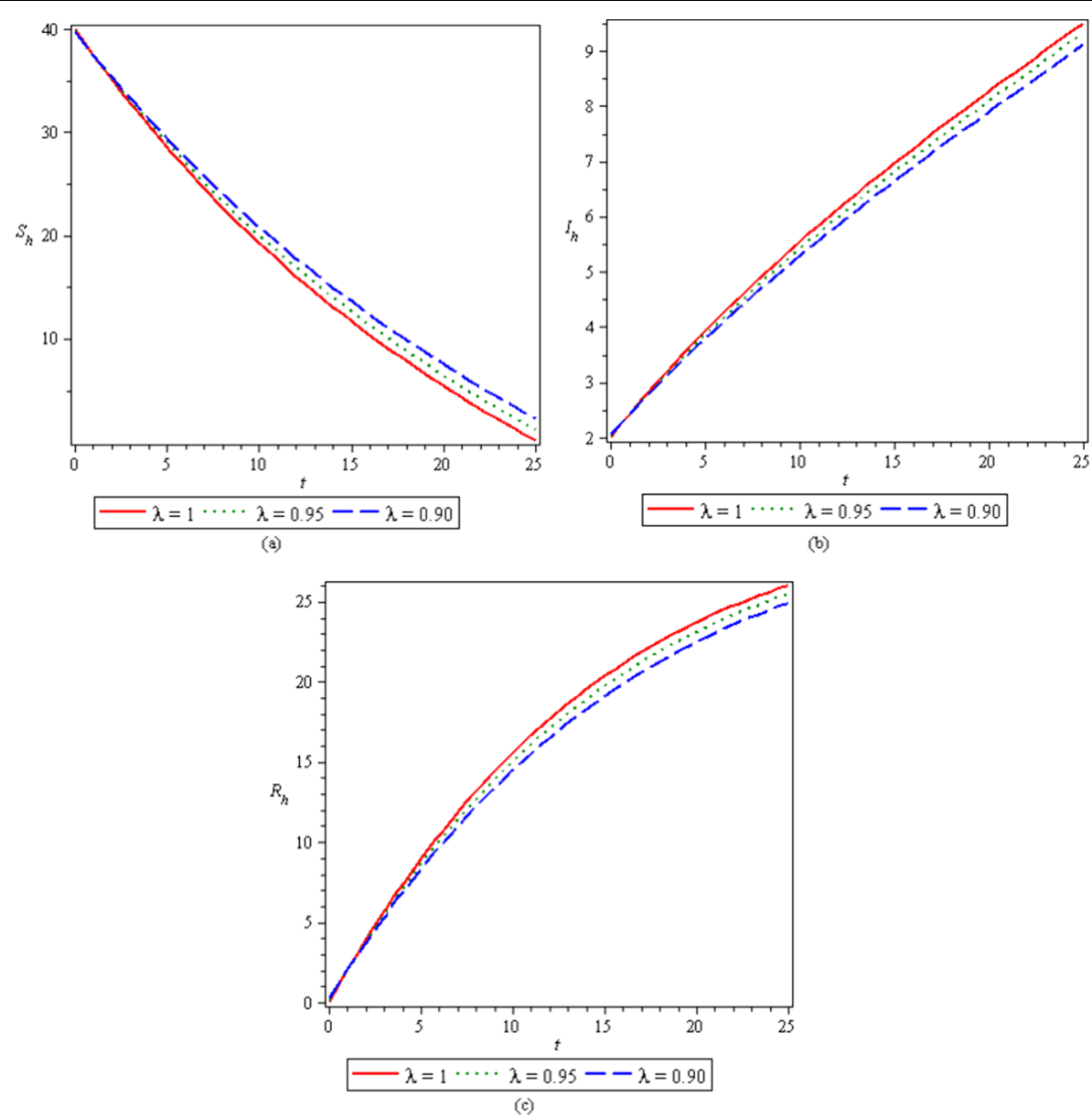

Figure 1 Effect of order of CF fractional derivative on human population when $\delta=0.05, v=0.05$, and $\sigma=0.05$ : (a) Susceptible humans, (b) Infected humans, (c) Recovered humans 

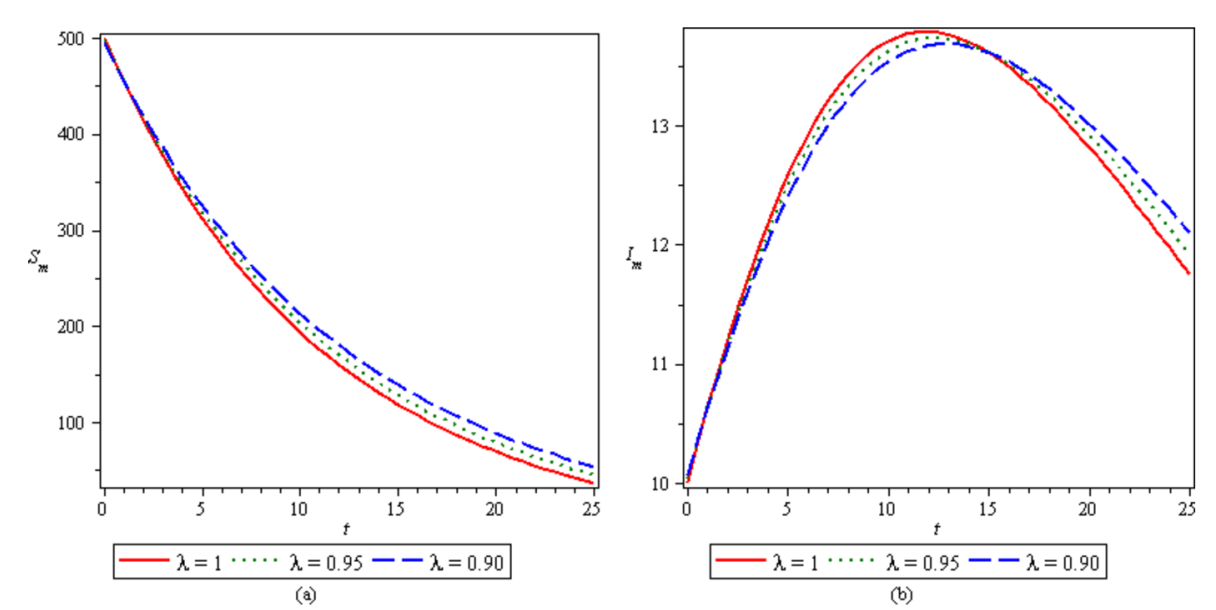

Figure 2 Effect of order of CF fractional derivative on mosquito population when $\delta=0.05, v=0.25$, and $\sigma=0.05$ : (a) Susceptible mosquito, (b) Infected mosquito
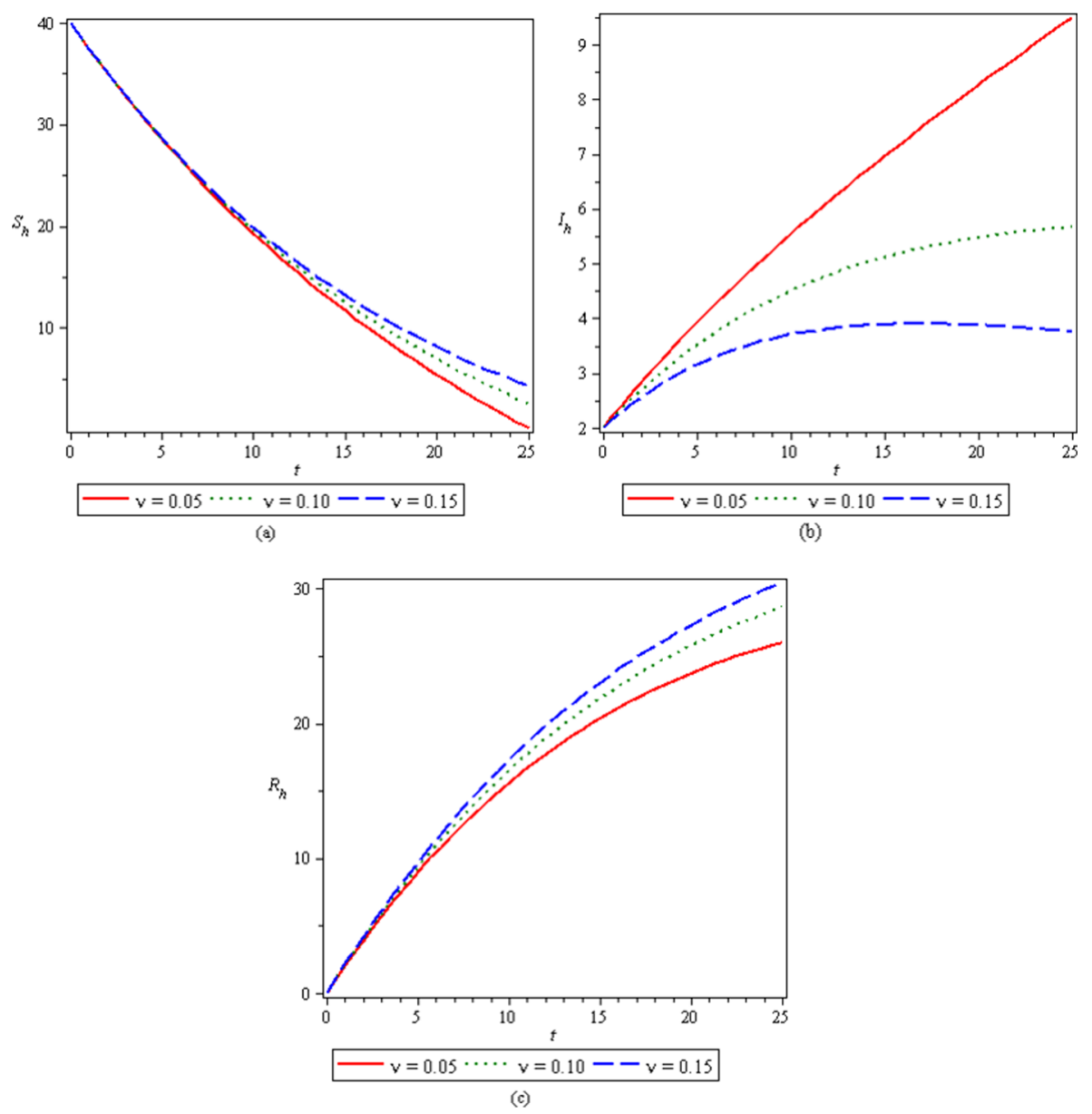

Figure 3 Effect of antimalarial drugs on human population when $\lambda=1, \delta=0.05$, and $\sigma=0.05$ : (a) Susceptible humans; (b), Infected humans, (c) Recovered humans 

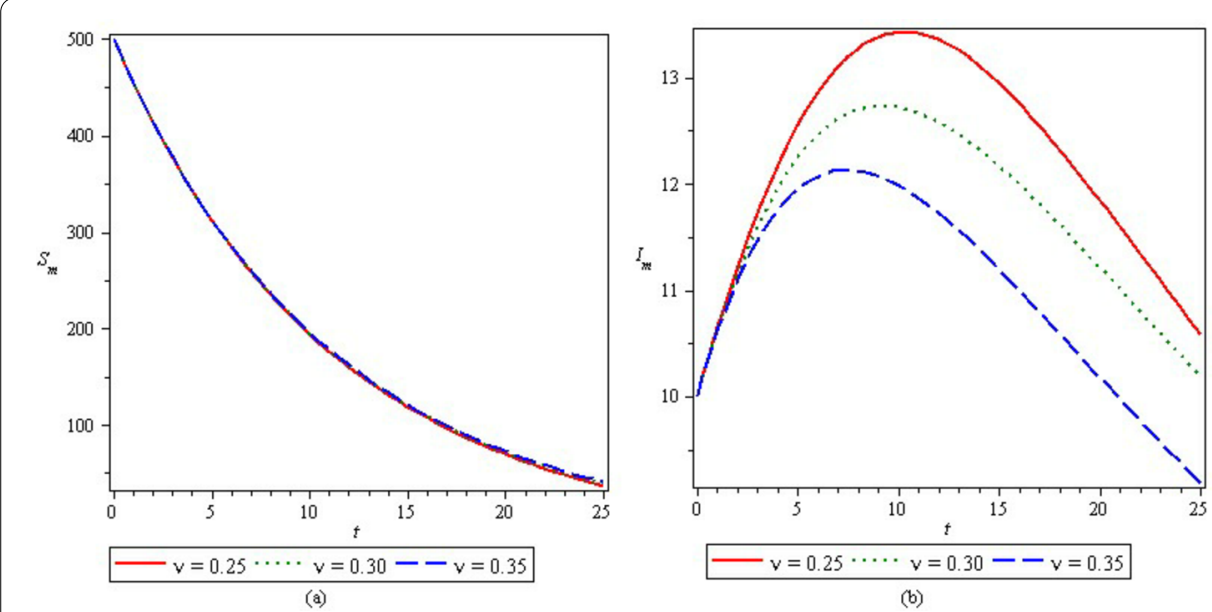

Figure 4 Effect of antimalarial drugs on mosquito population when $\lambda=1, \delta=0.05$, and $\sigma=0.05$ : (a) Susceptible mosquito, (b) Infected mosquito
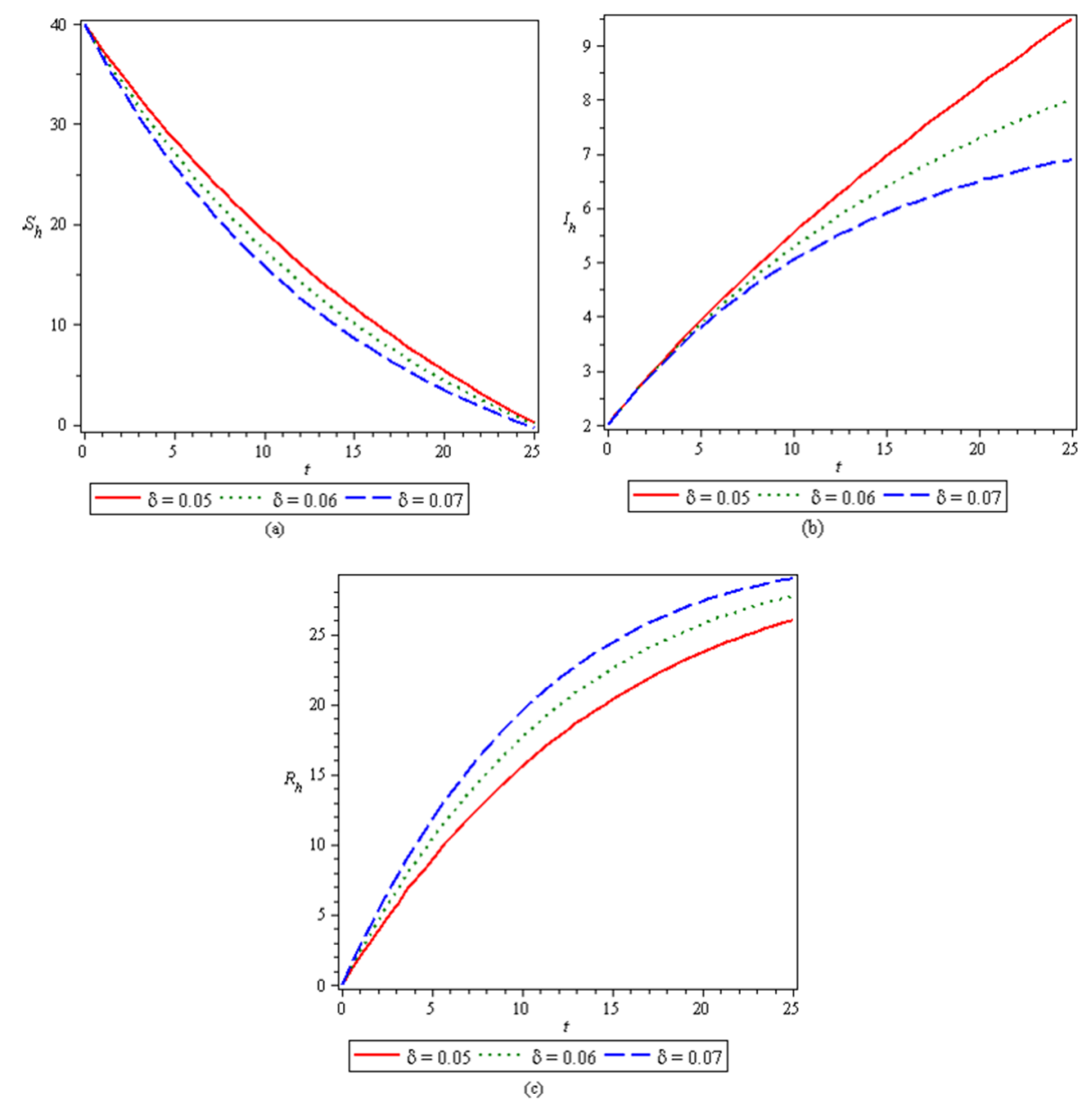

Figure 5 Effect of treatment of vaccines on human population when $\lambda=1, \nu=0.05$ and $\sigma=0.05$ : (a) Susceptible human, (b) Infected human, (c) Recovered human 

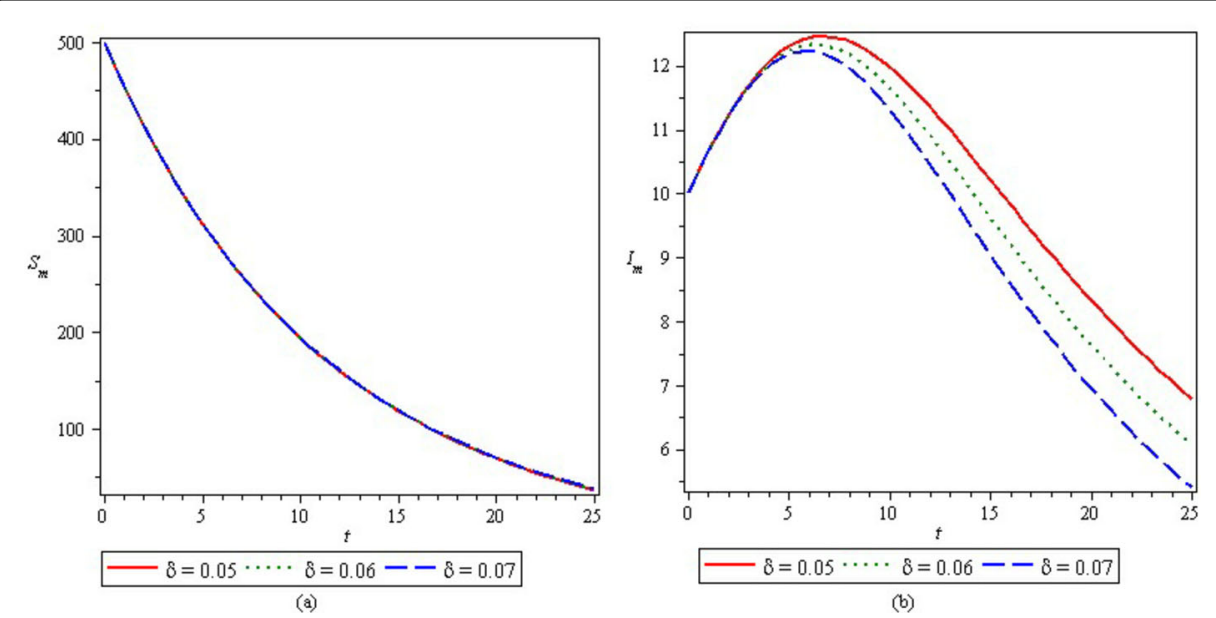

Figure 6 Effect of treatment of vaccines on mosquito population when $\lambda=1, v=0.25$, and $\sigma=0.05$ : (a) Susceptible mosquito (b) Infected mosquito
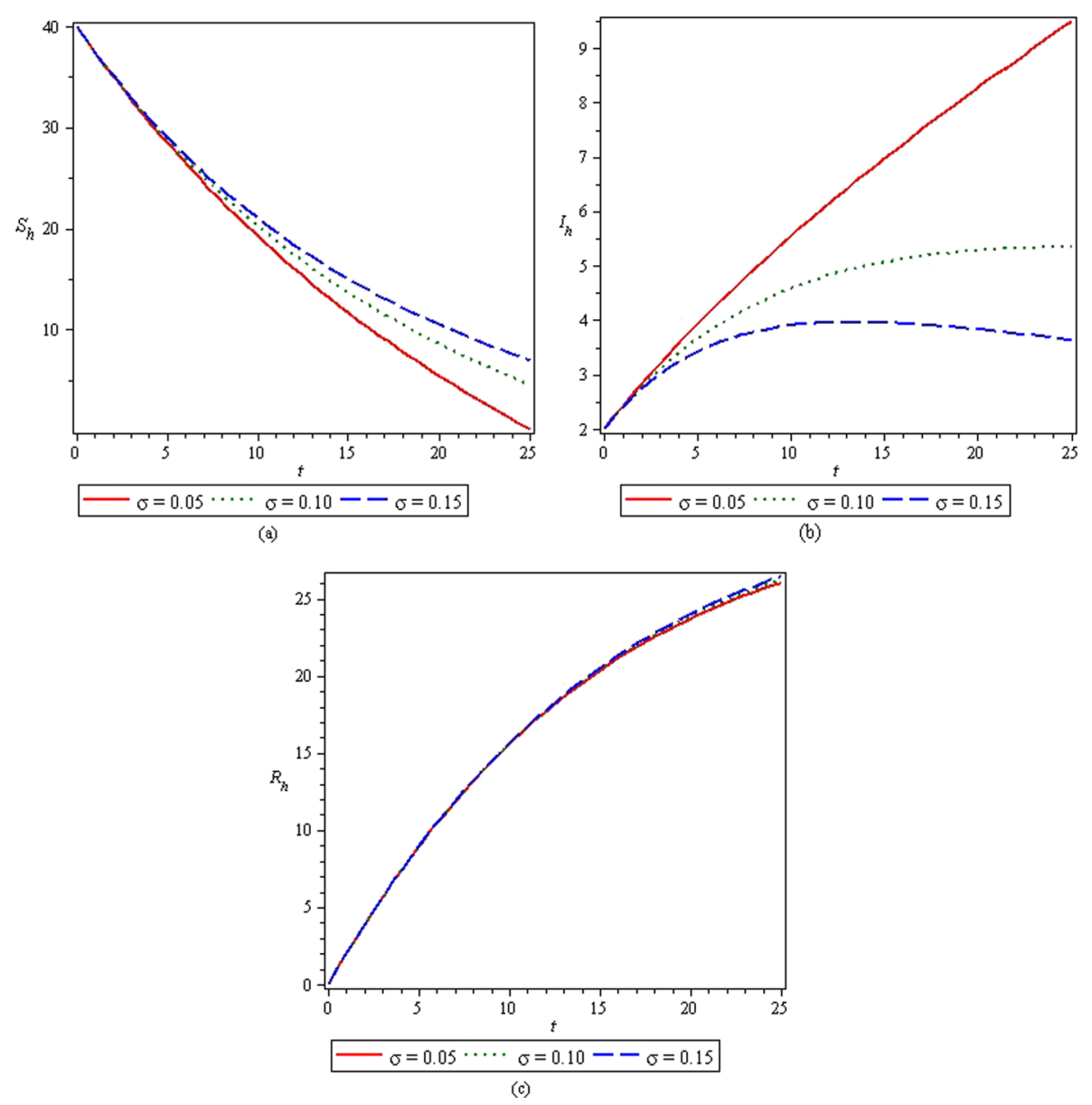

Figure 7 Effect of treatment of spraying on human population when $\lambda=1, v=0.05$ and $\delta=0.05$ : (a) Susceptible human, (b) Infected human, (c) Recovered human 

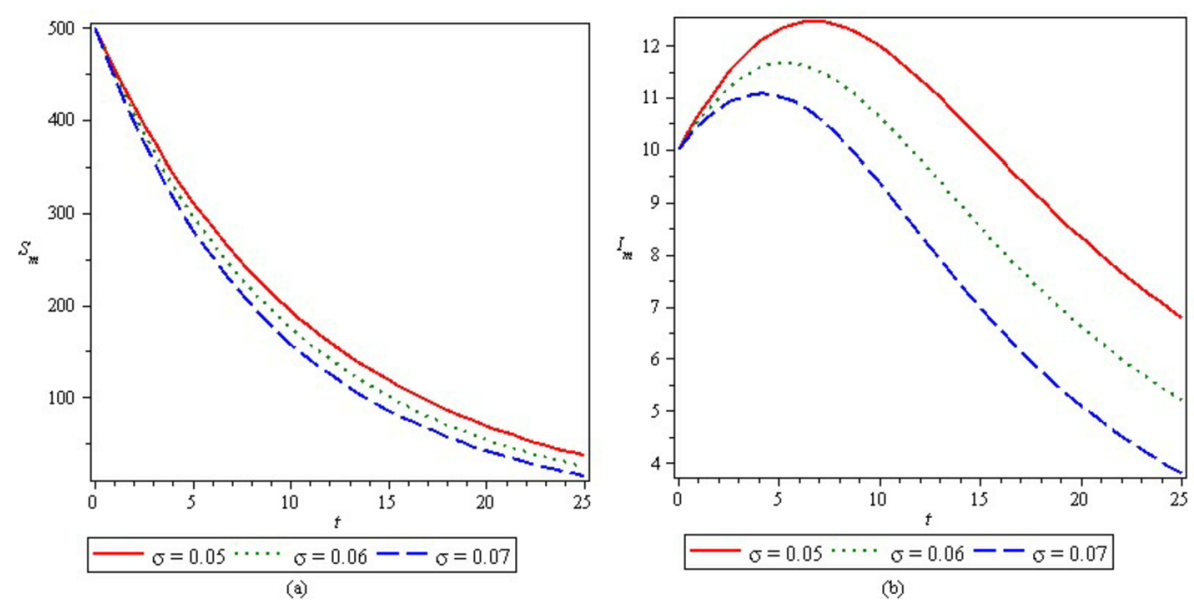

Figure 8 Effect of treatment of spraying on mosquito population when $\lambda=1, v=0.25$, and $\delta=0.05$ : (a) Susceptible mosquitos, (b) Infected mosquitos

antimalarial drugs on different classes of mosquito population. Figure 5 presents the influence of treatment of vaccines on various classes of human population. Figure 6 exhibits the effect of treatment of vaccines on various groups of mosquito population. In Fig. 7, the influence of treatment of spraying on various classes of human population is displayed. In Fig. 8, the impact of treatment of spraying on distinct classes of mosquito population is shown.

\section{Conclusions}

In this paper, we studied a fractional SIRS-SI malaria model transmission along with a number of cures such as the utilization of vaccines, antimalarial medicines, and spraying. The power of this model was inclusion of memory effects. The theory of fixed point was employed to examine the existence and uniqueness of solution of the considered fractional SIRS-SI model describing the spreading of malaria. The HATM and Padé approximation were applied to perform numerical simulation. The effects of order of the CF fractional derivative, vaccines, antimalarial drugs, and spraying on different groups of human populations and mosquito populations were analyzed. From the results we conclude that the $\mathrm{CF}$ fractional derivative is very useful for describing the treatment and control of the malaria disease and similar type of problems.

\section{Funding}

The authors extend their appreciation to the International Scientific Partnership Program ISPP at King Saud University for funding this research work through ISPP\# 63.

\section{Competing interests}

The authors declare that they have no competing interests.

\section{Authors' contributions}

DK, JS, MAQ, and DB designed the study, developed the methodology, collected the data, performed the analysis, and wrote the manuscript. All authors read and approved the final manuscript.

\section{Author details}

'Department of Mathematics, University of Rajasthan, Jaipur, India. ${ }^{2}$ Department of Mathematics, JECRC University, Jaipur, India. ${ }^{3}$ Department of Mathematics, College of Science, King Saud University, Riyadh, Saudi Arabia. ${ }^{4}$ Department of Mathematics, Faculty of Arts and Sciences, Cankaya University, Etimesgut, Turkey. ${ }^{5}$ Institute of Space Sciences, Magurele-Bucharest, Romania. 


\section{Publisher's Note}

Springer Nature remains neutral with regard to jurisdictional claims in published maps and institutional affiliations.

Received: 23 November 2018 Accepted: 14 June 2019 Published online: 09 July 2019

References

1. Agusto, F.B., Marcus, N., Okosun, K.O.: Application of optimal control to the epidemiology of malaria. Electron. J. Differ. Equ. 2012, 81 (2012)

2. Abdullahi, M.B., Hasan, Y.A., Abdullah, F.A.: A mathematical model of malaria and the effectiveness of drugs. Appl. Math. Sci. 7(62), 3079-3095 (2013)

3. Mandal, S., Sarkar, R.R., Sinha, S.: Mathematical models of malaria-a review. Malar. J. 10, 1-19 (2011)

4. Chiyaka, C., Tchuenche, J.M., Garira, W., Dube, S.: A mathematical analysis of the effects of control strategies on the transmission dynamics of malaria. Appl. Math. Comput. 195, 641-662 (2008)

5. Rafikov, M., Bevilacqua, L., Wyse, A.P.P.: Optimal control strategy of malaria vector using genetically modified mosquitoes. J. Theor. Biol. 258, 418-425 (2009)

6. Yang, H.M.: A mathematical model for malaria transmission relating global warming and local socioeconomic conditions. Rev. Saude Publica 35(3), 224-231 (2001)

7. Senthamarai, R., Balamuralitharan, S., Govindarajan, A.: Application of homotopy analysis method in SIRS-SI model of malaria disease. Int. J. Pure Appl. Math. 113(12), 239-248 (2017)

8. Caputo, M.: Elasticita e dissipazione. Zani-Chelli, Bologna (1969)

9. Kilbas, A.A., Srivastava, H.M., Trujillo, J.J.: Theory and Applications of Fractional Differential Equations. Elsevier, Amsterdam (2006)

10. Baleanu, D., Guvenc, Z.B., Machado, Z.A.T.: New Trends in Nanotechnology and Fractional Calculus Applications. Springer, Dordrecht (2010)

11. Yang, X.J., Machado, Z.A.T., Baleanu, D., Cattani, C.: On exact traveling-wave solutions for local fractional Korteweg-de Vries equation. Chaos 26, 084312 (2016)

12. Atangana, A., Alqahtani, R.T.: New numerical method and application to Keller-Segel model with fractional order derivative. Chaos Solitons Fractals 116, 14-21 (2018)

13. Singh, J., Kumar, D., Nieto, J.J.: Analysis of an El Nino-Southern Oscillation model with a new fractional derivative. Chaos Solitons Fractals 99, 109-115 (2017)

14. Kumar, D., Singh, J., Baleanu, D.: A new analysis of Fornberg-Whitham equation pertaining to a fractional derivative with Mittag-Leffler type kernel. Eur. Phys. J. Plus 133(2), 70 (2018)

15. Singh, J., Kumar, D., Baleanu, D., Rathore, S.: An efficient numerical algorithm for the fractional Drinfeld-Sokolov-Wilson equation. Appl. Math. Comput. 335, 12-24 (2018)

16. Singh, J., Kumar, D., Baleanu, D.: On the analysis of fractional diabetes model with exponential law. Adv. Differ. Equ. 2018, $231(2018)$

17. Pinto, C.M.A., Carvalho, A.R.M.: The role of synaptic transmission in a HIV model with memory. Appl. Math. Comput. 292, 76-95 (2017)

18. Pinto, C.M.A.: Persistence of low levels of plasma viremia and of the latent reservoir in patients under ART: a fractional-order approach. Commun. Nonlinear Sci. Numer. Simul. 43, 251-260 (2017)

19. Pinto, C.M.A., Carvalho, A.R.M.: Fractional complex-order model for HIV infection with drug resistance during therapy. J. Vib. Control (2015). https://doi.org/10.1177/1077546315574964

20. Magin, R.L.: Fractional Calculus in Bioengineering. Begell House Inc. Publishers, Redding (2006)

21. Atangana, A., Baleanu, D.: New fractional derivative with nonlocal and non-singular kernel, theory and application to heat transfer model. Therm. Sci. 20(2), 763-769 (2016)

22. Caputo, M., Fabrizio, M.: A new definition of fractional derivative without singular kernel. Prog. Fract. Differ. Appl. 1, 73-85 (2015)

23. Losada, J.J., Nieto, J.: Properties of the new fractional derivative without singular kernel. Prog. Fract. Differ. Appl. 1 , 87-92 (2015)

24. Singh, J., Kumar, D., Hammouch, Z., Atangana, A.: A fractional epidemiological model for computer viruses pertaining to a new fractional derivative. Appl. Math. Comput. 316, 504-515 (2018)

25. Singh, J., Kumar, D., Qurashi, M.A., Baleanu, D.: A new fractional model for giving up smoking dynamics. Adv. Differ. Equ. (2017). https://doi.org/10.1186/s13662-017-1139-9

26. Djida, J.D., Atangana, A.: More generalized groundwater model with space-time Caputo Fabrizio fractional differentiation. Numer. Methods Partial Differ. Equ. 33(5), 1616-1627 (2017)

27. Liao, S.J.: Beyond Perturbation: Introduction to Homotopy Analysis Method. Chapman and Hall / CRC Press, Boca Raton (2003)

28. Liao, S.J.: An approximate solution technique not depending on small parameters: a special example. Int. J. Non-Linear Mech. 30, 371-380 (1995)

29. Khan, M., Gondal, M.A., Hussain, I., Vanani, S.K.: A new comparative study between homotopy analysis transform method and homotopy perturbation transform method on semi-infinite domain. Math. Comput. Model. 55, $1143-1150$ (2012)

30. Kumar, D., Singh, J., Baleanu, D., Rathore, S.: Analysis of a fractional model of Ambartsumian equation. Eur. Phys. J. Plus $133,259(2018)$

31. Kumar, D., Singh, J., Baleanu, D.: A fractional model of convective radial fins with temperature-dependent thermal conductivity. Rom. Rep. Phys. 69(1), 103 (2017)

32. Boyd, J.P.: Padé approximants algorithm for solving nonlinear ordinary differential equation boundary value problems on an unbounded domain. Comput. Phys. 11, 299-303 (1997)

33. Atangana, A., Alkahtani, B.T.: Analysis of non-homogenous heat model with new trend of derivative with fractional order. Chaos Solitons Fractals 89, 566-571 (2016)

34. Atangana, A., Alqahtani, B.S.T.: Analysis of the Keller-Segel model with a fractional derivative without singular kernel. Entropy 17, 4439-4453 (2015)

35. Kumar, D., Singh, J., Baleanu, D.: Analysis of regularized long-wave equation associated with a new fractional operator with Mittag-Leffler type kernel. Physica A 492, 155-167 (2018) 